\title{
Do Fluid-Attenuated Inversion Recovery Vascular Hyperintensities Represent Good Collaterals before Reperfusion Therapy?
}

\author{
(D) E. Mahdjoub, (D) G. Turc, (D). Legrand, (D). Benzakoun, DM. Edjlali, DP. Seners, (D) S. Charron, DW. Ben Hassen, (D) O. Naggara, \\ (D).-F. Meder, (D).-L. Mas, (D).-C. Baron, and (DC. Oppenheim
}

\begin{abstract}
BACKGROUND AND PURPOSE: In acute ischemic stroke, whether FLAIR vascular hyperintensities represent good or poor collaterals remains controversial. We hypothesized that extensive FLAIR vascular hyperintensities correspond to good collaterals, as indirectly assessed by the hypoperfusion intensity ratio.

MATERIALS AND METHODS: We included 244 consecutive patients eligible for reperfusion therapy with MCA stroke and pretreatment MR imaging with both FLAIR and PWI. The FLAIR vascular hyperintensity score was based on ASPECTS, ranging from 0 (no FLAIR vascular hyperintensity) to 7 (FLAIR vascular hyperintensities abutting all ASPECTS cortical areas). The hypoperfusion intensity ratio was defined as the ratio of the time-to-maximum $>10$-second over time-to-maximum $>6$-second lesion volumes. The median hypoperfusion intensity ratio was used to dichotomize good (low hypoperfusion intensity ratio) versus poor (high hypoperfusion intensity ratio) collaterals. We then studied the association between FLAIR vascular hyperintensity extent and hypoperfusion intensity ratio.
\end{abstract}

RESULTS: Hypoperfusion was present in all patients, with a median hypoperfusion intensity ratio of 0.35 (interquartile range, $0.19-0.48$ ). The median FLAIR vascular hyperintensity score was 4 (interquartile range, 3-5). The FLAIR vascular hyperintensities were more extensive in patients with good collaterals (hypoperfusion intensity ratio $\leq 0.35$ ) than with poor collaterals (hypoperfusion intensity ratio $>0.35 ; P$ for Trend $=.016)$. The FLAIR vascular hyperintensity score was independently associated with good collaterals $(P$ for Trend $=.002)$.

CONCLUSIONS: In patients eligible for reperfusion therapy, FLAIR vascular hyperintensity extent was associated with good collaterals, as assessed by the pretreatment hypoperfusion intensity ratio. The ASPECTS assessment of FLAIR vascular hyperintensities could be used to rapidly identify patients more likely to benefit from reperfusion therapy.

ABBREVIATIONS: FVH = FLAIR vascular hyperintensity; HIR = hypoperfusion intensity ratio; IQR = interquartile range; Tmax $=$ time-to-maximum

C ollateral circulation plays an important role in stroke pathophysiology. ${ }^{1}$ In response to a middle cerebral artery occlusion, flow in leptomeningeal anastomoses from anterior and posterior cerebral arteries reverts and pial collaterals dilate, providing blood supply to maintain tissue viability. Assessing collateral status has direct applications for decision-making and predicting outcome after acute ischemic stroke. Using collateral-based imaging as an entry selection criterion, a recent trial showed that rapid endovascular treatment dramatically improved functional

Received March 17, 2017; accepted after revision August 24.

From the Departments of Radiology (E.M., L.L., J.B., M.E., S.C., W.B.H., O.N., J.-F.M., C.O.) and Neurology (G.T., P.S., J.-L.M., J.-C.B.), Université Paris Descartes, Institut national de la santé et de la recherche médicale S894, Département HospitaloUniversitaire Neurovasc, Centre Hospitalier Sainte-Anne, Paris, France.

Please address correspondence to Catherine Oppenheim, MD, PhD, CH SainteAnne, 1 rue Cabanis, 75014 Paris, France; e-mail: c.oppenheim@ch-sainte-anne.fr

三 Indicates article with supplemental on-line table.

http://dx.doi.org/10.3174/ajnr.A5431 outcome in patients with proximal vessel occlusion, small infarct core, and moderate-to-good collateral circulation. ${ }^{2}$ DSA remains the reference to assess collateral status, ${ }^{1}$ but less invasive methods based on postcontrast $\mathrm{CT}^{2}$ or MR imaging ${ }^{3,4}$ have been developed. On the basis of first-pass gadolinium PWI, the hypoperfusion intensity ratio (HIR) has been creatively proposed to assess the severity of hypoperfusion. ${ }^{5}$ Defined as the proportion of the time-to-maximum (Tmax) $>10$-second over the Tmax $>6$-second lesion volumes, the HIR is a good predictor of collateral status, as demonstrated by correlations with DSA: A low HIR corresponded to good collaterals and predicted smaller infarct growth and better clinical outcome than a high HIR. ${ }^{5,6}$

A means to assess collateral status without the need for contrast agent, additional scanning time, or automated perfusion software would be desirable for patient management. FLAIR, which is part of most stroke MR imaging protocols, provides information about vessel status. High signal intensities within blood vessels, in subarachnoid spaces, distal to arterial occlusion, ${ }^{7}$ 
termed FLAIR vascular hyperintensities (FVHs), ${ }^{8,9}$ are related to hemodynamic impairment and represent slow retrograde flow in leptomeningeal collaterals. ${ }^{10}$ However, whether they reflect good or poor collaterals remains controversial. For some authors, FVHs represent poor collaterals and predict larger infarct growth $^{11,12}$ and worse clinical outcome. ${ }^{12-14}$ In the only study using the HIR to assess collaterals, extensive FVHs, assessed dichotomously as FVHs visible in $>4$ axial sections, were associated with high HIR (ie, poor collaterals) in 62 patients with arterial occlusion in any territory on prethrombolysis MRA. ${ }^{12}$ Conversely, other studies found that FVHs indicate good collaterals, with extensive FVHs associated with smaller baseline DWI lesions, ${ }^{15-18}$ less severe neurologic deficits, ${ }^{15-17}$ smaller infarct growth, ${ }^{16,18,19}$ and better clinical outcome. ${ }^{15,18}$ We previously reported similar findings based on FVH presence beyond the boundaries of the cortical DWI lesion in patients with proximal MCA occlusion, ${ }^{9}$ which also predicted a better clinical response to recanalization. ${ }^{20}$ This accumulated evidence indirectly suggests that FVHs represent good collaterals. Accordingly, we aimed to determine whether extensive FVHs are associated with good collaterals, using HIR as a surrogate marker for collaterals, in a large cohort of patients with MCA territory stroke eligible for reperfusion therapy.

\section{MATERIALS AND METHODS}

This retrospective study was based on a prospectively collected monocentric register of consecutive (2003-2015) patients treated by intravenous thrombolysis and/or thrombectomy. The inclusion criteria were acute ischemia in the MCA territory; pretreatment MR imaging with DWI, FLAIR, and PWI; and 24-hour follow-up MR imaging to assess infarct growth. Patients with anterior cerebral artery or posterior circulation strokes were excluded because the ASPECTS was originally designed for the MCA territory. Patients with severe artifacts on DWI, FLAIR, or PWI were also excluded. Early neurologic improvement was defined as a $\geq 8$-point decrease in the NIHSS score within the first 24 hours or an NIHSS score $\leq 1$ at 24 hours. Excellent or favorable outcomes were defined as 3-month $\mathrm{mRS} \leq 1$ or $\leq 2$, respectively. In accordance with French legislation, ethics committee approval was not required because our study only implied retrospective analysis of anonymized data collected as part of routine clinical care.

MR imaging has been systematically implemented in our center as first-line diagnostic imaging in candidates for reperfusion therapies. Pretreatment and 24-hour follow-up MR imaging were performed on a $1.5 \mathrm{~T}$ scanner (Signa; GE Healthcare, Milwaukee, Wisconsin) using a standardized protocol, ${ }^{9}$ including DWI, FLAIR, T2*, intracranial 3D-TOF MRA, and an additional PWI sequence for pretreatment MR imaging. FLAIR parameters were TR/TE/TI, 8277-9802/155.5-159.4/2093-2300 ms; FOV, $24 \times 24$ $\mathrm{cm}^{2}$; matrix, $256 \times 192 ; 1$ excitation; 24 contiguous axial sections, 6-mm thick; 2-minute18-second maximal duration. PWI consisted of a $2^{*}$-weighted echo-planar sequence with TR/TE, 2000/60 ms; FOV, $24 \times 24 \mathrm{~cm}^{2}$; matrix, $64 \times 96$; 1 excitation; repetition, 50 times after a bolus $(5-7 \mathrm{~mL} / \mathrm{s})$ of $20 \mathrm{~mL}$ of gadoteric acid.

FVHs were defined as focal, tubular, or serpentine hyperinten- sities in subarachnoid spaces with a typical arterial course. ${ }^{8}$ Blinded to PWI and clinical data, we semiquantitatively assessed FVHs using the FVH score, according to their spatial distribution in the 7 ASPECTS cortical areas (insula, M1-M6). ${ }^{9}$ An ASPECTS cortical area was considered positive when it coincided with an FVH. The FVH score ranged from 0 (no FVH) to 7 (FVHs abutting all ASPECTS cortical areas). The FVH score was additionally rated by a second reader in half of the population.

BrainStat arterial input function software (READY View; GE Healthcare) was used to generate Tmax maps. An automated 3D rigid registration (FMRIB Linear Image Registration Tool, FLIRT, Version 5.5; http://www.fmrib.ox.ac.uk/fsl/fslwiki/FLIRT) between Tmax maps and DWI was performed and manually corrected whenever necessary. MANGO software (Version 3.8; Research Imaging Institute, UTHSCSA; http://ric.uthscsa.edu/ mango/) was used to successively extract a brain mask of ADC $<$ $1.3 \times 10^{-3} \mathrm{~mm}^{2} / \mathrm{s}$ to remove CSF voxels, project it onto Tmax maps, and extract brain voxels with Tmax $>6$ seconds and $>10$ seconds. HIR was computed as the ratio between the Tmax $>10$ second over Tmax $>6$-second volumes. Pretreatment $\left(\mathrm{DWI}_{1}\right)$ and follow-up $\left(\mathrm{DWI}_{2}\right)$ volumes were manually segmented using interactive tools based on DWI signal intensity. ${ }^{21}$ Infarct growth was defined as the difference between $\mathrm{DWI}_{2}$ and $\mathrm{DWI}_{1}$ volumes. A PWI-DWI mismatch was considered present when Tmax $>6$ second volume exceeded $1.8 \times \mathrm{DWI}_{1}$ volume. ${ }^{6}$ The occlusion site was categorized into proximal (internal carotid artery and/or M1 segment of the MCA) or distal. Complete recanalization was defined as an arterial occlusive lesion score of 3 on 24-hour MRA.

The intraclass correlation coefficient was used to assess interobserver agreement for the FVH score. For further analyses, the FVH score was considered in 4 categories (ASPECTS $=0-1,2-3$, $4-5$, or $6-7$ ) to avoid small numbers in extreme FVH scores. The median HIR in our cohort was used to dichotomize good (low HIR) versus poor (high HIR) collaterals. ${ }^{6,12}$ A univariable analysis comparing patients with low and high HIRs was used to test our hypothesis that extensive FVHs represent good collaterals (Student $t$ or Mann-Whitney $U$ test for continuous variables; $\chi^{2}$ or the Fisher exact test for categoric variables, as appropriate; and the Cochran-Armitage test for ordinal variables). Considering potential collinearity, baseline variables with a $P$ value $<.10$ in the univariable analysis were entered into a multivariable binary logistic regression model with a dichotomized HIR as the dependent variable. All the above analyses were repeated using the median HIR threshold of 0.4, derived from the Diffusion Weighted Imaging Evaluation for Understanding Stroke Evolution 2 (DEFUSE 2) cohort and found to be a good predictor of collateral status. ${ }^{6}$ In an additional univariable analysis, we further compared patients according to FVH extent for clinical and imaging data, 24-hour infarct growth, early neurologic improvement, and 3-month mRS $\left(\chi^{2}\right.$, Mann-Whitney $U$, or Jonckheere-Terpstra test, as appropriate). A $P$ value $<.05$ was considered significant (SPSS, Version 19, IBM, Armonk, New York; SAS 9.4, SAS Institute, Cary, North Carolina).

\section{RESULTS}

During the study period, 777 patients underwent intravenous thrombolysis and/or thrombectomy; 533 patients were excluded 
Table 1: Comparison of patients with low and high $\mathrm{HIR}^{\mathrm{a}}$

\begin{tabular}{|c|c|c|c|}
\hline Characteristics & $\begin{array}{l}\text { Low HIR } \\
(n=122)\end{array}$ & $\begin{array}{l}\text { High HIR } \\
(n=122)\end{array}$ & $\begin{array}{c}P \\
\text { Value }\end{array}$ \\
\hline Age & $70(56-79)$ & $71(60-79)$ & .446 \\
\hline Male (No.) (\%) & $55(45)$ & $83(68)$ & $<.001$ \\
\hline Hypertension (No.) (\%) & $68(57)$ & $66(55)$ & .685 \\
\hline Diabetes mellitus (No.) (\%) & $12(10)$ & 20 (17) & .142 \\
\hline Hyperlipidemia (No.) (\%) & $31(26)$ & $40(33)$ & .251 \\
\hline Current smoking (No.) (\%) & $23(20)$ & $25(21)$ & .771 \\
\hline Systolic BP (mm Hg) & 149 (139-163) & $156(139-173)$ & .141 \\
\hline Diastolic BP (mm Hg) & $82(71-89)$ & 81 (70-93) & .990 \\
\hline Serum glucose $(\mathrm{mmol} / \mathrm{L})^{d}$ & $6.1(5.4-7.0)$ & $6.5(5.5-7.9)$ & .038 \\
\hline NIHSS ${ }^{b}$ score & $11(7-17)$ & $16(11-21)$ & $<.001$ \\
\hline \multicolumn{4}{|l|}{ Baseline MRI } \\
\hline Onset-to-MRI time (min) & $112(86-153)$ & $112(84-156)$ & .838 \\
\hline Proximal occlusion (No.) (\%) & $81(66)$ & $80(66)$ & .893 \\
\hline $\mathrm{DWI}_{1}$ volume $(\mathrm{mL})$ & $8(4-19)$ & $41(16-96)$ & $<.001$ \\
\hline Tmax $>6$-sec volume $(\mathrm{mL})$ & $44(17-85)$ & $115(56-147)$ & $<.001$ \\
\hline Tmax $>10$-sec volume (mL) & $9(2-22)$ & $52(27-75)$ & $<.001$ \\
\hline PWI-DWI mismatch (No.) (\%) & $85(70)$ & $59(48)$ & $<.001$ \\
\hline FVH score (No.) (\%) & & & $.016^{\mathrm{e}}$ \\
\hline $0-1$ & $6(5)$ & $13(11)$ & \\
\hline $2-3$ & $30(25)$ & $33(27)$ & \\
\hline $4-5$ & $54(44)$ & $59(48)$ & \\
\hline $6-7$ & $32(26)$ & $17(14)$ & \\
\hline \multicolumn{4}{|l|}{ 24-hr follow-up } \\
\hline NIHSS score ${ }^{f}$ & $6(2-15)$ & $14(7-20)$ & $<.001$ \\
\hline ENI (No.) $(\%)^{f}$ & $51(42)$ & $27(22)$ & .001 \\
\hline $\mathrm{DWI}_{2}$ volume (Ml) & $18(9-45)$ & $87(33-161)$ & $<.001$ \\
\hline Infarct growth (Ml) & $6(1-26)$ & $29(10-75)$ & $<.001$ \\
\hline Complete recanalization (No.) (\%) & $40(33)$ & $45(37)$ & .502 \\
\hline $3-\mathrm{mo}$ mRS $\leq 1(\mathrm{No}.)(\%)^{\mathrm{g}}$ & $49(45)$ & $25(21)$ & $<.001$ \\
\hline $3-\mathrm{mo} m R S \leq 2(\mathrm{No})(\%)^{\mathrm{g}}$ & $63(58)$ & $51(44)$ & .033 \\
\hline
\end{tabular}

Note:-BP indicates blood pressure; ENI, early neurologic improvement.

${ }^{a}$ Unless specified, numbers are median (interquartile range).

${ }^{\mathrm{b}}$ Missing data for 4 patients.

cMissing data for 9 patients.

${ }^{\mathrm{d}}$ Missing data for 13 patients.

e $P$ for Trend.

${ }^{\mathrm{f}}$ Missing data for 5 patients.

${ }^{g}$ Missing data for 18 patients.

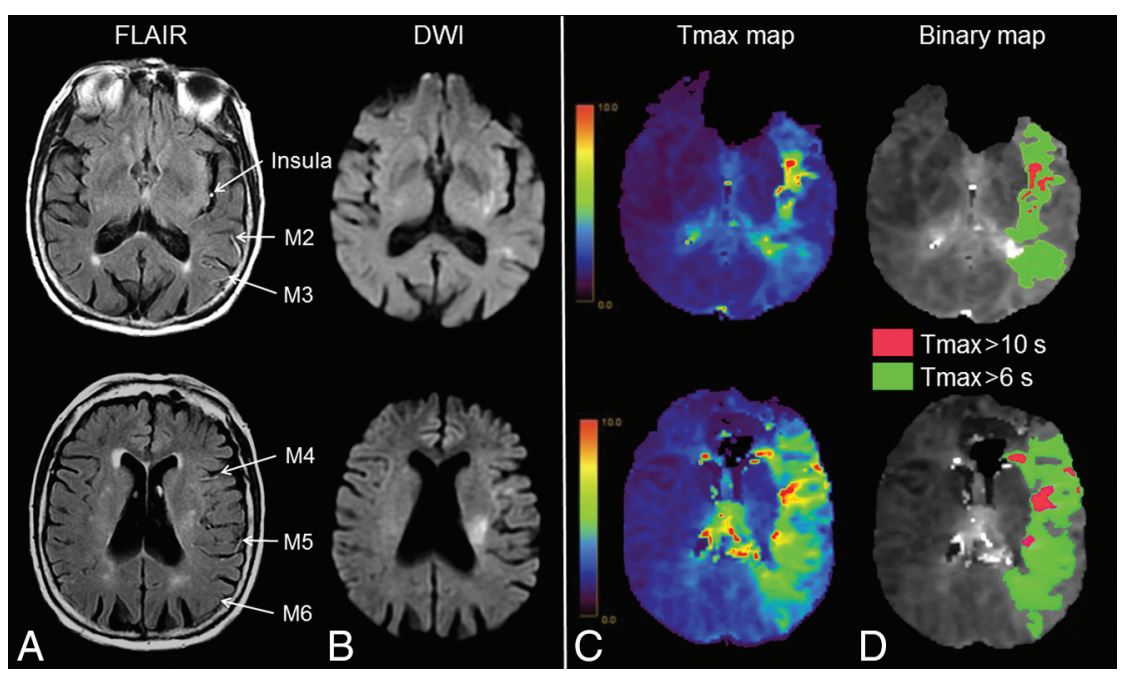

FIG 1. Extensive FVHs and low hypoperfusion intensity ratio. MR imaging obtained 110 minutes after stroke onset (NIHSS score = 12) in an 88-year-old woman. A, FVHs in 6 ASPECTS areas (insula, M2-M6), corresponding to a 6-point FVH-score. $B$, Thirteen-milliliter diffusion-weighted imaging lesion. Tmax map (C) with 85-mL Tmax $>6$-second lesion and 15-mL Tmax $>10$-second lesion (D) $(\mathrm{HIR}=0.18)$. After intravenous thrombolysis, the 24-hour NIHSS score was 1, and the DWI lesion was $12 \mathrm{~mL}$ (not shown). The 3-month mRS was 1 . (pretreatment CT scan, $n=85$; pretreatment MR imaging not available in DICOM format, $n=49$; PWI not performed, $n=272$; severe artifacts on DWI, FLAIR, or PWI, $n=26$; non-MCA stroke, $n=84$; no follow-up MR imaging, $n=14$; or pretreatment 3T MR imaging, $n=3$ ). They did not differ from the 244 included patients as to sex $(P=$ $.45)$, age $(P=.46)$, or baseline NIHSS scores $(P=.19)$. Two-hundred eight $(85.3 \%)$ patients were treated by intravenous thrombolysis; 23 (9.4\%), by thrombectomy; and 13 (5.3\%), by bridging therapy. Median time to treatment was 150 minutes (interquartile range [IQR], 120-189) for thrombolysis and 230 minutes (IQR, 149-401) for thrombectomy. One hundred sixty-one (66\%) patients had a proximal occlusion (internal carotid artery, $n=47$, or M1, $n=114)$.

Interobserver agreement for the FVH score was excellent (intraclass correlation coefficient, 0.88 ; $95 \% \mathrm{CI}, 0.84-$ 0.91). The median FVH score in the whole population was 4 (IQR, 3-5). FVHs were abutting the insula, M2, M3, M5, M1, M6, and M4 ASPECTS areas in 240 (98\%), 209 (86\%), 156 (64\%), 150 (61\%), 126 (52\%), $125(51 \%)$, and 59 (24\%) patients. Hypoperfusion was present in all patients with median Tmax $>6$-second and $>10$-second volumes of $74 \mathrm{~mL}$ (minimum, 0.95; maximum, 258) and $24 \mathrm{~mL}$ (minimum, 0.06; maximum, 160), respectively. The median HIR was 0.35 (IQR, 0.19-0.48).

In univariable analysis (Table 1), patients with good collaterals (HIR $\leq 0.35$, Fig 1) had higher FVH scores ( $P$ for Trend $=.016)$ than those with poor collaterals (HIR $>0.35$, Fig 2). Patients with good collaterals also had lower baseline NIHSS scores, smaller $\mathrm{DWI}_{1}$ volumes, smaller Tmax $>10$-second and Tmax $>6$-second volumes, and more frequently had a PWI-DWI mismatch $(P<.001)$. In multivariable analysis (Table 2), the FVH score remained significantly associated with a low HIR $(P$ for Trend $=.002)$, after adjustment of potential confounders. Results were similar using the alternative HIR threshold of $0.4^{6,22}$ for dichotomization between good and poor collaterals (data not shown). 


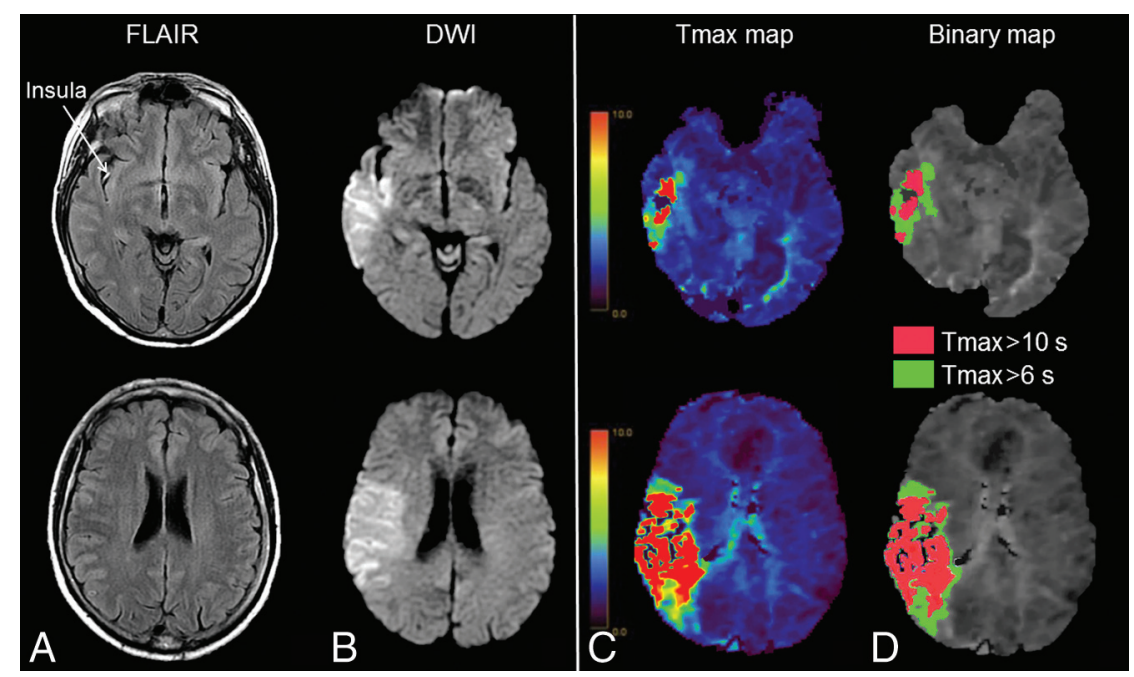

FIG 2. Few FVHs and high hypoperfusion intensity ratio. MR imaging obtained 180 minutes after stroke onset (NIHSS score $=12$ ) in a 56-year-old man. A, FVHs in the insula, corresponding to a 1-point FVH score. B, Ninety-four milliliter diffusion-weighted imaging lesion. Tmax map (C) with 91-mL Tmax >6-second lesion and 53-mL Tmax >10-second lesion (D) (HIR = 0.58). After intravenous thrombolysis, the 24-hour NIHSS score was 14, and the DWI lesion was $150 \mathrm{~mL}$ (not shown). The 3-month mRS was 3 .

Table 2: Factors associated with a low HIR in multivariable analysis

\begin{tabular}{|c|c|c|}
\hline Characteristics & $\begin{array}{l}\text { Adjusted OR } \\
(95 \% \mathrm{CI})\end{array}$ & $\begin{array}{c}P \\
\text { Value }\end{array}$ \\
\hline Male & $0.98(0.42-2.29)$ & .953 \\
\hline $\begin{array}{l}\text { Serum glucose, per 1-mmol/L } \\
\text { increase }\end{array}$ & $0.99(0.83-1.18)$ & .882 \\
\hline $\begin{array}{l}\text { Baseline NIHSS score, per 1-point } \\
\text { increase }^{\text {b }}\end{array}$ & $1.04(0.96-1.12)$ & .343 \\
\hline $\mathrm{DWI}_{1}$ volume, per 1-mL increase & $1.01(0.99-1.03)$ & .446 \\
\hline $\begin{array}{l}\text { Tmax }>10-\text { sec volume, per 1-mL } \\
\text { increase }\end{array}$ & $0.89(0.85-0.92)$ & $<.001$ \\
\hline PWI-DWI mismatch & $8.95(2.65-30.22)$ & $<.001$ \\
\hline FVH-score & - & $.002^{\mathrm{d}}$ \\
\hline $0-1$ & 1 & - \\
\hline $2-3$ & $1.17(0.25-5.50)$ & .844 \\
\hline $4-5$ & $2.39(0.56-10.22)$ & .242 \\
\hline $6-7$ & $34.20(4.35-268.60)$ & .001 \\
\hline
\end{tabular}

a Missing data for 13 patients.

${ }^{\mathrm{b}}$ Missing data for 4 patients.

'Tmax $>6$ sec was not selected with $T \max >10$ sec to avoid collinearity.

d $P$ for Trend.

When we compared patients according to their FVH scores (Table 3), patients with extensive FVHs were imaged earlier $(P=$ $.007)$ and had more proximal occlusions $(P<.001)$, more PWIDWI mismatch $(P=.011)$, and lower HIR, considered as a continuous variable $(P=.049)$. Despite similar baseline NIHSS scores and recanalization rates, patients with extensive FVHs tended to have smaller infarct growth $(P=.125)$ and more frequently experienced an early neurologic improvement $(P<.001)$ or an excellent 3 -month mRS $(P=.039)$ than those with fewer FVHs.

To rule out the potential influence of the occlusion site on our main findings, we analyzed post hoc the subset of 161 proximal occlusions, with similar results in univariable analysis (On-line Table), and again found an independent association between a high FVH score and a low HIR in multivariable analysis $(P$ for Trend $=.031)$. Of note, when we compared patients with proxi- mal occlusion according to their FVH scores, patients with extensive FVHs had smaller DWI volumes $(P=.003)$ and higher DWI-ASPECTS scores $(P=$ $.006)$ on pretreatment MR imaging.

\section{DISCUSSION}

In a large population of patients with MCA stroke who underwent MR imaging with PWI before reperfusion therapy, we found the following: 1) FVH extent was independently associated with a low HIR (ie, proportionally milder hypoperfusion and hence good collaterals); and 2) patients with extensive FVHs more frequently experienced early neurologic improvement and excellent 3month outcome, despite similar initial stroke severity and recanalization rates. These findings strengthen the notion that extensive FVHs represent a reliable surrogate for good collaterals, which maintain the viability of the ischemic tissue while awaiting reperfusion.

The presence of FVHs is considered a reliable marker of arterial occlusion ${ }^{7,13,23-25}$ and, consequently, is associated with more severe admission neurologic deficits than its absence. Like others, ${ }^{17}$ we found FVHs in most patients (99\%) with arterial occlusion. Besides their dichotomous presence or absence, FVH extent has been quantitated with various methods. In one method, FVHs were rated as involving less than or more than one-third of the MCA territory or the hypoperfused area, ${ }^{16,19}$ but determining this cutoff is notoriously difficult. FVHs have also been graded according to their sulcal location ${ }^{26}$ or by counting their number, ${ }^{11}$ which is cumbersome in the hyperacute clinical setting. Finally, counting axial FLAIR sections with FVHs has recently been proposed, ${ }^{12}$ but this does not account for the actual extent within each section and depends on the section number and thickness. We, like others, have previously assessed FVH topography relative to the DWI lesion, considering their rostrocaudal and anteroposterior distributions, but this approach depends on the size of the DWI lesion and does not yield the total extent of all FVHs. ${ }^{9,17,20}$ Our simple approach overcomes most of these limitations, affords an excellent interobserver concordance, and improves feasibility relative to manually counting all FVHs for clinical implementation.

Only a few studies have focused on the significance of FVH extent in the early time window. One study did not find an association between FVH extent and clinical outcome, ${ }^{25}$ while several others reported that extensive FVHs were associated with smaller baseline DWI lesions, ${ }^{9,15-17}$ larger PWI-DWI mismatch, ${ }^{9,16}$ smaller infarct growth, ${ }^{16,18,19}$ and better clinical outcome. ${ }^{15,17,18}$ Similarly, we showed an independent association between extensive FVHs and a low HIR (ie, good collaterals). Patients with extensive FVHs also had a larger PWI-DWI mismatch, suggesting larger penumbra due to robust collaterals protecting the penumbra from rapidly decaying while awaiting reperfusion. Accord- 


\begin{tabular}{|c|c|c|c|c|c|}
\hline & \multicolumn{4}{|c|}{ FVH Score } & \multirow[b]{2}{*}{$P$ Value } \\
\hline & $0-1(n=19)$ & $2-3(n=63)$ & $4-5(n=113)$ & $6-7(n=49)$ & \\
\hline Age (yr) & $73(60-80)$ & $70(57-79)$ & $71(56-80)$ & $67(62-77)$ & .339 \\
\hline Male (No.) (\%) & $12(63)$ & $31(49)$ & $68(60)$ & $27(55)$ & .500 \\
\hline Hypertension (No.) (\%) ${ }^{\mathrm{b}}$ & $12(63)$ & $38(60)$ & $58(51)$ & $26(53)$ & .537 \\
\hline Diabetes mellitus (No.) (\%) & $4(21)$ & $9(14)$ & $13(12)$ & $6(12)$ & .708 \\
\hline Hyperlipidemia (No.) (\%) & $9(47)$ & $18(29)$ & $43(38)$ & $18(37)$ & .477 \\
\hline Current smoking (No.) (\%) ${ }^{c}$ & $3(16)$ & $12(19)$ & $23(20)$ & $10(20)$ & .954 \\
\hline Systolic BP (mm Hg) & 148 (140-172) & $159(142-173)$ & $151(139-167)$ & $148(134-160)$ & .071 \\
\hline Diastolic BP (mm Hg) & $87(76-93)$ & $84(69-95)$ & $80(70-89)$ & $80(72-90)$ & .334 \\
\hline Serum glucose $(\mathrm{mmol} / \mathrm{L})^{d}$ & $6.6(5.5-8.1)$ & $6.7(5.4-7.3)$ & $6.4(5.5-7.6)$ & $6.0(5.3-7.0)$ & .189 \\
\hline Baseline NIHSS score ${ }^{b}$ & $13(8-20)$ & $13(7-18)$ & $15(8-20)$ & $15(11-21)$ & .321 \\
\hline Onset-to-MRI time (min) & $142(84-196)$ & $115(89-176)$ & $115(91-151)$ & $97(83-130)$ & .007 \\
\hline Proximal occlusion (No.) (\%) & $6(31)$ & $31(49)$ & $81(72)$ & $43(88)$ & $<.001$ \\
\hline $\mathrm{DWI}$, volume $(\mathrm{mL})$ & $24(12-102)$ & $19(5-67)$ & $18(7-49)$ & $12(6-34)$ & .077 \\
\hline Tmax $>10$-sec volume $(\mathrm{mL})$ & $26(5-76)$ & $17(4-43)$ & $24(11-53)$ & $30(11-64)$ & .200 \\
\hline Tmax $>6$-sec volume (mL) & $65(19-133)$ & $47(19-102)$ & $78(40-123)$ & $96(66-134)$ & .002 \\
\hline$H I R \leq 0.35(\mathrm{No}).(\%)$ & $6(32)$ & $30(48)$ & $54(47)$ & $32(65)$ & .057 \\
\hline HIR & $0.44(0.30-0.54)$ & $0.39(0.19-0.48)$ & $0.33(0.19-0.47)$ & $0.32(0.14-0.44)$ & .049 \\
\hline PWI-DWI mismatch (No.) (\%) & $6(32)$ & $32(51)$ & $71(63)$ & $35(51)$ & .011 \\
\hline $\mathrm{DWI}_{2}$ volume $(\mathrm{mL})$ & $73(14-150)$ & $43(13-147)$ & $37(13-104)$ & $29(12-52)$ & .075 \\
\hline 24-hr infarct growth (mL) & $27(2-87)$ & $19(4-57)$ & $14(1-45)$ & $13(4-28)$ & .125 \\
\hline Complete recanalization (No.) (\%) & $7(37)$ & $19(30)$ & $38(34)$ & $21(43)$ & .551 \\
\hline ENI (No.) (\%) & $7(37)$ & $12(20)$ & $30(27)$ & $29(53)$ & $<.001$ \\
\hline $3-\mathrm{mo}$ mRS $\leq 1$ (No.) $(\%)^{\mathrm{f}}$ & $3(17)$ & $14(24)$ & $35(34)$ & $22(45)$ & .039 \\
\hline $3-\mathrm{mo} m R S \leq 2(\mathrm{No})(\%)^{\mathrm{f}}$ & $9(47)$ & $26(41)$ & $47(42)$ & $32(65)$ & .057 \\
\hline
\end{tabular}

Note:-ENI indicates early neurologic improvement; BP, blood pressure.

${ }^{a}$ Unless specified, numbers are median (interquartile range).

${ }^{\mathrm{b}}$ Missing data for 4 patients.

${ }^{c}$ Missing data for 9 patients.

${ }^{\mathrm{d}}$ Missing data for 13 patients.

e Missing data for 5 patients.

${ }^{\mathrm{f}}$ Missing data for 18 patients.

ingly, extensive FVHs were associated with smaller ischemic lesions before treatment in patients with proximal occlusion, reinforcing our hypothesis that FVHs represent good collaterals.

The benefit of FVH-ASPECTS over simple DWI-ASPECTS for the assessment of collateral status remains to be evaluated. Most important, patients with extensive FVHs more frequently experienced early neurologic improvement and excellent 3-month outcome in contrast to those with fewer FVHs, despite more proximal occlusions and similar recanalization rates. Conversely, our findings stand in apparent contradiction to other studies suggesting that FVHs represent poor collaterals. 7,12,13,23,24 However, these studies either dichotomously categorized patients into the presence or absence of $\mathrm{FVHs}^{7,13,23}$ rather than analyzing $\mathrm{FVH}$ extent and/or were conducted in patients not eligible for thrombolysis. ${ }^{24}$ These differences might explain the discrepancy with our findings. Perhaps more surprising are the results from Kufner et al, ${ }^{12}$ who found that FVHs rather reflected poor collaterals using the HIR in patients with arterial occlusion before thrombolysis. This discrepancy might result from several differences between this study and ours. First, Kufner et al used a 3T scanner compared with 1.5T here, and the significance of FVHs may depend on magnetic field strength and sequence parameters. ${ }^{27} \mathrm{Sec}-$ ond, we studied a larger and more homogeneous population of overall severe anterior circulation strokes, as opposed to mixed anterior and posterior milder strokes in the Kufner et al study. The unreported proportion of posterior circulation strokes might have influenced their results because the reliability of HIR as a surrogate for good-versus-poor collaterals in this stroke subtype is unknown. Third, Kufner et al dichotomized their sample according to whether FVHs were visible over at least four 5-mm thick FLAIR sections, and such a cutoff might not have equal meaning for anterior-versus-posterior circulation strokes. Fourth, they merged patients with M1, M2, P1, P2, and vertebral occlusions into a single "medium vessel occlusion" group with an unreported proportion of distal occlusions.

FVHs might, however, be a marker for proximal occlusion ${ }^{23}$ rather than an indicator of collateral flow. Distal occlusions are indeed unlikely to result in extensive FVHs, irrespective of the quality of collaterals. However, the independent association between the FVH score and the low HIR we found in the population mixing distal and proximal occlusions remained significant in the 161 patients with proximal occlusion. This strengthens the link between FVH visibility and the quality of collaterals, irrespective of the occlusion type. Finally, the definition of HIR differed between our study and that of Kufner et al ${ }^{12}$ (Tmax 10/6 seconds here versus Tmax $8 / 2$ seconds), but the definition we used predicted rates of collateral flow, infarct growth, and clinical outcome in the DEFUSE 2 cohort. $^{6}$ Further studies are needed to determine which of the above differences accounts best for the discrepancy between the 2 studies.

Direct comparisons between FVH extent and DSA are limited. ${ }^{10,15,26,28}$ Except from a seminal study in which only 8 patients underwent DSA, ${ }^{7}$ others consistently reported FVHs to be associated with good collaterals. ${ }^{10,15,26,28}$ Our results are consistent with the latter DSA-FLAIR correlations. Although the HIR is an indirect marker of collateral status, ${ }^{5,6}$ PWI is obtained within 
minutes of FLAIR, thereby overcoming the issue of the delay between MR imaging and DSA inherent in all MR imaging-DSA comparisons. Given the transient nature of $\mathrm{FVHs},{ }^{7}$ correlation between MR images obtained nearly simultaneously provides new insights into the debated significance of FVHs.

That numerous well-developed collaterals appear as extensive FVHs deserves attention. In ischemic stroke, FVHs are undoubtedly related to retrograde collateral flow, which reaches cortical areas later than anterograde flow, often during the venous angiographic phase. This sluggish flow likely explains, at least in part, FVH visibility. ${ }^{10}$ A recent study showed that FVHs were more prominent when the arterial circulation time on DSA was neither too short ( $>1$ second) nor too long ( $<7.98$ seconds) ${ }^{28}$ Given that prominent FVHs are observed in a wide range of intermediate time delays of retrograde collateral filling, ${ }^{28} \mathrm{FVH}$ visibility might depend on not only flow speed but also the amount of recruitable collaterals. Extensive FVHs could reflect the recruitment of numerous well-developed collaterals from both the anterior cerebral artery and posterior cerebral arteries (which can cover the entire ischemic bed). FVHs abutting anterosuperior ASPECTS areas may correspond to leptomeningeal collaterals from the anterior cerebral artery to the MCA, and FVHs abutting posteroinferior ASPECTS areas may correspond to leptomeningeal collaterals from the posterior cerebral artery to the MCA. ${ }^{26}$

This retrospective study has several limitations. First, collateral status was indirectly based on the HIR. The validity of the dichotomization at the median to categorize into good and poor collaterals can also be questioned. Our median HIR was, however, identical to that observed in the study of Bang et al. ${ }^{5}$ We also checked that our results were not affected by using the median HIR value of 0.4 derived from the DEFUSE 2 cohort. ${ }^{6}$ Second, despite similar recanalization rates in patients with low/high HIRs or in those with few/extensive FVHs, we cannot exclude some patients having futile recanalization because the latter was assessed on the 24-hour MRA. Third, our results are based on 1.5T MR imaging data from the same manufacturer with standardized parameters. Although this ensures data homogeneity, it prevents extrapolating to other magnetic fields, coil systems, or FLAIR parameters that may influence FVH visibility. ${ }^{27}$ Fourth, we collapsed FVH data as a single score, assuming that each ASPECTS area had the same significance. However, FVHs in different ASPECTS locations may not share the same pathophysiology. ${ }^{15,17,24,26}$ Further studies are needed to determine the implications of FVH location, if any. Last, most patients were treated by thrombolysis alone, following guidelines at the time they had a stroke. Although the association between the FVH score and HIR is independent of the type of treatment, the association with outcome might differ in patients treated according to current state-of-the-art guidelines. Because we found that extensive FVHs represented good collaterals, their link with early neurologic improvement and favorable outcome could be stronger if recanalization rates increase, as expected with bridging therapy.

\section{CONCLUSIONS}

Using quantitative assessment of FVHs and the HIR as a marker of collateral status, we found a significant association between FVH extent and good collaterals in patients with MCA stroke before reperfusion therapy. Thus, to rapidly identify patients more likely to benefit from reperfusion therapy, ASPECTS assessment of FVHs might serve as a surrogate for collateral status whenever perfusion data or fast postprocessing software is not available.

\section{REFERENCES}

1. McVerry F, Liebeskind DS, Muir KW. Systematic review of methods for assessing leptomeningeal collateral flow. AJNR Am J Neuroradiol 2012;33:576-82 CrossRef Medline

2. Goyal M, Demchuk AM, Menon BK, et al; ESCAPE Trial Investigators. Randomized assessment of rapid endovascular treatment of ischemic stroke. N Engl J Med 2015;372:1019-30 CrossRef Medline

3. Campbell BC, Christensen S, Tress BM, et al; EPITHET Investigators. Failure of collateral blood flow is associated with infarct growth in ischemic stroke. J Cereb Blood Flow Metab 2013;33:1168-72 CrossRef Medline

4. Kim SJ, Son JP, Ryoo S, et al. A novel magnetic resonance imaging approach to collateral flow imaging in ischemic stroke. Ann Neurol 2014;76:356-69 CrossRef Medline

5. Bang OY, Saver JL, Alger JR, et al; UCLA Collateral Investigators. Determinants of the distribution and severity of hypoperfusion in patients with ischemic stroke. Neurology 2008;71:1804-11 CrossRef Medline

6. Olivot JM, Mlynash M, Inoue M, et al; DEFUSE 2 Investigators. Hypoperfusion intensity ratio predicts infarct progression and functional outcome in the DEFUSE 2 cohort. Stroke 2014;45:1018-23 CrossRef Medline

7. Kamran S, Bates V, Bakshi R, et al. Significance of hyperintense vessels on FLAIR MRI in acute stroke. Neurology 2000;55:265-69 CrossRef Medline

8. Azizyan A, Sanossian N, Mogensen MA, et al. Fluid-attenuated inversion recovery vascular hyperintensities: an important imaging marker for cerebrovascular disease. AJNR Am J Neuroradiol 2011; 32:1771-75 CrossRef Medline

9. Legrand L, Tisserand M, Turc G, et al. Do FLAIR vascular hyperintensities beyond the DWI lesion represent the ischemic penumbra? AJNR Am J Neuroradiol 2015;36:269-74 CrossRef Medline

10. Sanossian N, Saver JL, Alger JR, et al. Angiography reveals that fluidattenuated inversion recovery vascular hyperintensities are due to slow flow, not thrombus. AJNR Am J Neuroradiol 2009;30:564-68 CrossRef Medline

11. Hohenhaus M, Schmidt WU, Brunecker P, et al. FLAIR vascular hyperintensities in acute ICA and MCA infarction: a marker for mismatch and stroke severity? Cerebrovasc Dis 2012;34:63-69 CrossRef Medline

12. Kufner A, Galinovic I, Ambrosi V, et al. Hyperintense vessels on FLAIR: hemodynamic correlates and response to thrombolysis. AJNR Am J Neuroradiol 2015;36:1426-30 CrossRef Medline

13. Ebinger M, Kufner A, Galinovic I, et al. Fluid-attenuated inversion recovery images and stroke outcome after thrombolysis. Stroke 2012;43:539-42 CrossRef Medline

14. Girot M, Gauvrit JY, Cordonnier C, et al. Prognostic value of hyperintense vessel signals on fluid-attenuated inversion recovery sequences in acute cerebral ischemia. Eur Neurol 2007;57:75-79 Medline

15. Huang X, Liu W, Zhu W, et al. Distal hyperintense vessels on FLAIR: a prognostic indicator of acute ischemic stroke. Eur Neurol 2012;68: 214-20 CrossRef Medline

16. Lee KY, Latour LL, Luby M, et al. Distal hyperintense vessels on FLAIR: an MRI marker for collateral circulation in acute stroke? Neurology 2009;72:1134-39 CrossRef Medline

17. Liu D, Scalzo F, Rao NM, et al. Fluid-attenuated inversion recovery vascular hyperintensity topography, novel imaging marker for revascularization in middle cerebral artery occlusion. Stroke 2016;47: 2763-69 CrossRef Medline

18. Olindo S, Chausson N, Joux J, et al. Fluid-attenuated inversion recovery vascular hyperintensity: an early predictor of clinical out- 
come in proximal middle cerebral artery occlusion. Arch Neurol 2012;69:1462-68 CrossRef Medline

19. Pérez de la Ossa N, Hernández-Perez M, Domènech $S$, et al. Hyperintensity of distal vessels on FLAIR is associated with slow progression of the infarction in acute ischemic stroke. Cerebrovasc Dis 2012; 34:376-84 CrossRef Medline

20. Legrand L, Tisserand M, Turc G, et al. Fluid-attenuated inversion recovery vascular hyperintensities-diffusion-weighted imaging mismatch identifies acute stroke patients most likely to benefit from recanalization. Stroke 2016;47:424-27 CrossRef Medline

21. Hassen WB, Tisserand M, Turc G, et al. Comparison between voxelbased and subtraction methods for measuring diffusion-weighted imaging lesion growth after thrombolysis. Int J Stroke 2016;11: 221-28 CrossRef Medline

22. Wouters A, Dupont $P$, Christensen S, et al. Association between time from stroke onset and fluid-attenuated inversion recovery lesion intensity is modified by status of collateral circulation. Stroke 2016; 47:1018-22 CrossRef Medline

23. Cheng B, Ebinger M, Kufner A, et al; Stroke Imaging Repository (STIR) Investigators. Hyperintense vessels on acute stroke fluid- attenuated inversion recovery imaging: associations with clinical and other MRI findings. Stroke 2012;43:2957-61 CrossRef Medline

24. Kono T, Naka H, Nomura E, et al. The association between hyperintense vessel sign and final ischemic lesion differ in its location. $J$ Stroke Cerebrovasc Dis 2014;23:1337-43 CrossRef Medline

25. Schellinger PD, Chalela JA, Kang DW, et al. Diagnostic and prognostic value of early MR imaging vessel signs in hyperacute stroke patients imaged $<3$ hours and treated with recombinant tissue plasminogen activator. AJNR Am J Neuroradiol 2005;26: 618-24 Medline

26. Liu W, Xu G, Yue X, et al. Hyperintense vessels on FLAIR: a useful non-invasive method for assessing intracerebral collaterals. Eur J Radiol 2011;80:786-91 CrossRef Medline

27. Ahn SJ, Lee KY, Ahn SS, et al. Can FLAIR hyperintense vessel (FHV) signs be influenced by varying MR parameters and flow velocities? A flow phantom analysis. Acta Radiol 2016;57:580-86 CrossRef Medline

28. Lee SH, Seo KD, Kim JH, et al. Correlation between hyperintense vessels on FLAIR imaging and arterial circulation time on cerebral angiography. Magn Reson Med Sci 2016;15:105-10 CrossRef Medline 